The Korean Journal of Parasitology

Vol. 41, No. 2. 135-137, June 2003

\title{
Comparison of carbohydrate moieties of sparganum proteins of the snake, mouse and those of adult worm
}

\author{
Hyun Jong $\mathrm{YANG}^{*}$ \\ Department of Parasitology, College of Medicine, Ewha Womans University, Seoul, 158-710, Korea
}

\begin{abstract}
The carbohydrate moieties of larval sparganum proteins in two different species, the snakes, Elaphe rufodorsata, the Balb/c mouse and those of the adult worm, Spirometra erinacei, were compared using five different lectins including GNA, SNA, MAA, PNA and DSA. The GNA positive $53 \mathrm{kDa}$ molecule, which is excretory-secretory protease in the sparganum from the snake showed a stage specific and developmental regulation. We also suggested that sparganum glycosylation may be involved in immune evasion and differentiation into an adult worm.
\end{abstract}

Key words: sparganum, lectin, glycoprotein

Human sparganosis is a larval cestode disease caused by the ingestion of unfiltered water that is contaminated from procercoid or of plerocercoid in the reptile host. Despite the deleterious immune attacks from the host, the sparganum survives in the human being for a long time. A series of investigations have been focused on the identification of the surface or of the excretory-secretory molecules of the parasite in order to understand the basis of protective immunity (Kong et al., 1994, 1997). Several factors responsible for such a protection have been proposed. For example, plausible roles of cysteine proteases in immune evasion have been reported. These proteins are mostly glycoproteins in nature that bear various carbohydrate moieties by which they are able to possess the potential for a wide range of functional capacities. In this study, carbohydrate moieties of the glycoproteins of the soluble extract of

- Received 21 April 2003, accepted after revision 23 May 2003.

- This work was supported by Korea Research Foundation Grant (KRF-2001-041-F00077).

*Corresponding author (e-mail: parayang@ewha.ac.kr) the sparganum from each of the two species and adult Spirometra erinacei were observed using different types of specific lectins.

The spargana were collected from snakes Elaphe rufodorsata. The spargana were used to infect the experimental mouse and the worms were harvested 4 weeks after infection. The worms were homogenized with Teflon Pestle Homogenizer and centrifuged at $15,000 \mathrm{rpm}$ for $1 \mathrm{~h}$ at $4^{\circ} \mathrm{C}$. The supernatant was regarded as each sparganum extracts from snake and mouse. Adult worms were collected from small intestine of cats, which had been infected for 1 month with sparganum collected from snake. Crude extracts of the adult worm were prepared as described above. These extracts were stored at $-70^{\circ} \mathrm{C}$ until use.

Lectin bindings were examined with DIG Glycan Differentiation Kit (Roche Molecular Biochemicals, Germany). Lectins used in the study included Galanthus nivalis agglutinin (GNA), Sambucus nigra agglutinin (SNA), Maackia amurensis agglutinin (MAA), peanut agglutinin (PNA), Datura stramonium agglutinin (DSA). Each lectin exhibited a specific binding GNA to mannose, SNA to sialic acid linked 
$\alpha(2-6)$ to galactose, MAA to sialic acid linked $\alpha(2-3)$ to galactose, PNA to galactose- $\beta(1-3)-\mathrm{N}$-acetylgalactosamine and DSA to galactose- $\beta(1-4)-\mathrm{N}$-acetylglucosamine. Positive controls used were carboxypeptidase $Y$ for GNA, transferrin for SNA, fetuin for SNA, MAA and DSA and asilofetuin for PNA and DSA. The lectin blots were done according to the manufacturer's instruction. In brief, the proteins were separated by $7.5-15 \%$ sodium dodecyl sulfate (SDS)-PAGE and then transferred to a polyvinyl difluoride (PVDF) membrane. The transferred membrane was reacted with each digoxigenin labeled lectin at each required dilution, followed by an incubation with antidigoxigenin-alkaline phosphatase. The membranes were developed with NBT/X-phosphatase solution.

Analysis of the crude extracts of the larval sparganum and the adult worms is shown in Fig. 1A with Coomassie blue staining. The analysis of glycoproteins which were obtained from lectin blotting using different glycoconjugates is shown in
Fig. 1B. Lectins are very useful in identifying carbohydrate structure because they have a high affinity for specific glycoconjugates. The molecular weights of the glycoproteins identified by lectin blotting ranged from 10 to $180 \mathrm{kDa}$. Mannose epitopes are shown to be dominant carbohydrate moieties on the glycoproteins in the sparganum as indicated by strong staining with GNA lectin. This result is in agreement with the previous finding which showed that N-linked oligosaccharides were the main components of the parasite proteins (Gerard 1990; Hounsell 1994). The 27 and $53 \mathrm{kDa}$ proteins in the snake and mouse spargana may have N-linked glycans with carbohydrate chains of different compositions as shown in Fig. 1B (panel a). The 53 $\mathrm{kDa}$ protein was shown to bear high mannose or a hybrid type in its carbohydrate structure. The $53 \mathrm{kDa}$ molecule was not reactive with GNA in the crude extracts of the mouse spargana, which suggested that $53 \mathrm{kDa}$ molecule may be developmentally regulated

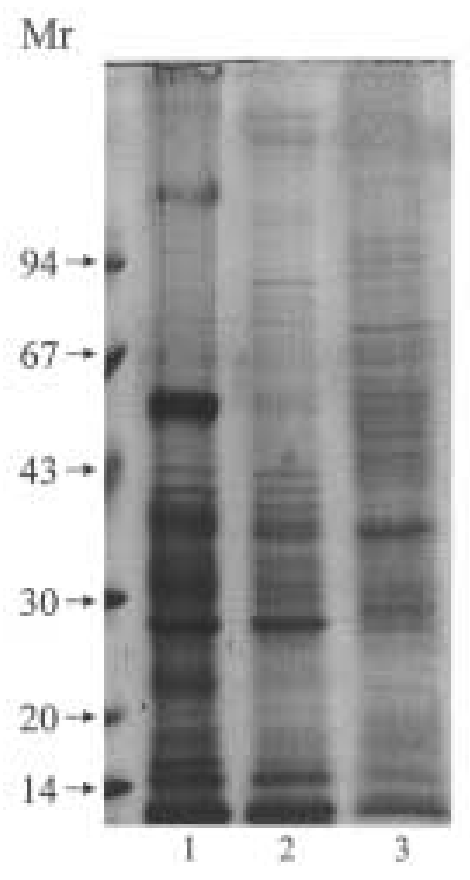

A
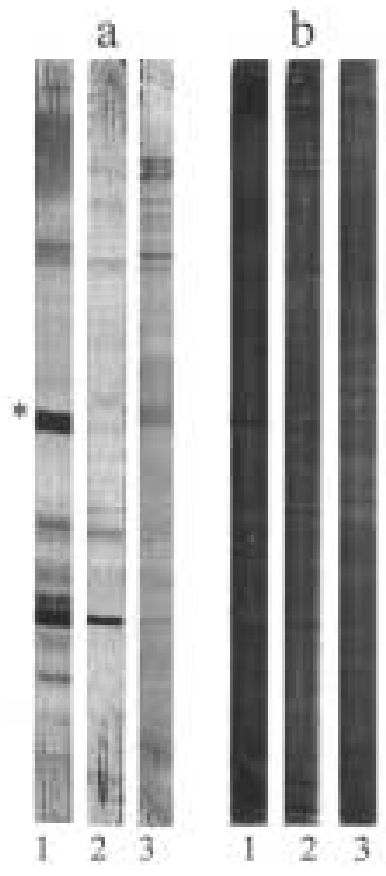

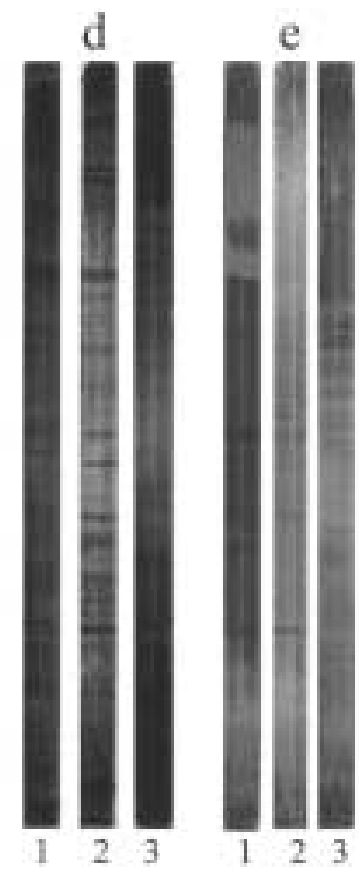

B

Fig. 1. Analysis of the crude extracts of the sparganum and adult worm by $7.5-15 \%$ SDS-PAGE (A) and lectin blot (B). Lectin blot of transferred membrane of (1) snake, (2) mouse sparganum and (3) adult worm probed with specific lectins: (a) Galanthus nivalis agglutinin (GNA), (b) Sambucus nigra agglutinin (SNA), (c) Maackia amurensis agglutinin (MAA), (d) peanut agglutinin (PNA), (e) Datura stramonium agglutinin (DSA). Asterisk $\left({ }^{*}\right)$ marked at 53 kDa shows that the protein is not reactive with crude extracts of mouse sparganum. All molecular weights are measured in kDa. 
similar to swine nodular worm Oesophagostomum dentatum (Joachim et al., 2001). However, the $27 \mathrm{kDa}$ protein exhibited positive reactions with all the glycoconjugate examined in the larval stage. This result suggested that $27 \mathrm{kDa}$ proteins have various glycan structures and may be involved in crucial roles of worm survival in the host.

The excretory-secretory products of Trichinella spiralis were composed of heavily-glycosylated molecules. These molecules were shown to be associated with local immune regulation during the infection (Jarvis and Pritchard, 1992; GrudenMovsesijan et al., 2002). In this respect, it was reported that expression of the $27 \mathrm{kDa}$ molecule of the sparganum was developmentally regulated from larval stage to adult stage (Kong et al., 2000). This finding was similar to the lectin blots in this study. It suggests that highly active excretory-secretory molecules of the sparganum may be associated with the immune evasion mechanisms of the worm from the host immune attack. Fewer faint reactive proteins were stained on the crude extracts of adult $S$. erinacei (Fig. 1B), which showed that $\mathrm{N}$ - or O-linked glycoproteins are more abundant in the larval sparganum than in adult stage. In the GNA positive $53 \mathrm{kDa}$ molecule, an excretory-secretory protease that was specifically expressed in the snake sparganum may also be developmentally regulated. For further research we could investigate whether the specific glycan structures of the sparganum protein play a critical role in protective immunity and in worm differentiation.

\section{REFERENCES}

Gerard C (1990) Purification of glycoproteins. Methods Enzymol 182: 529-539.

Gruden-Movsesijan A, Ilic N, Sofronic-Milosavljevic L (2002) Lectin-blot analyses of Trichinella spiralis muscle larvae excretory-secretory components. Parasitol Res 88: 1004-1007.

Hounsell EF (1994) Chracterization of the glycosylation status of proteins. Mol Biotechnol 2: 45-60.

Jarvis LM, Pritchard DI (1992) An evaluation of the role of carbohydrate epitopes in immunity to Trichinella spiralis. Parasite Immunol 14: 489-501.

Joachim A, Ruttkowski B, Daugschies A (2001) Characterization of stage-specific proteins of Oesophagostomum dentatum by preparative isoelectric focusing and lectin blotting. Parasitol Int 50: 41-45.

Kong Y, Chung YB, Cho SY, Kang SY (1994) Cleavage of immunoglobulin $\mathrm{G}$ by excretory-secretory cathepsin Slike protease of Spirometra mansoni plerocercoid. Parasitology 109: 611-621.

Kong Y, Kang SY, Kim SH, Chung YB, Cho SY (1997) A neutral cysteine protease of Spirometra mansoni plerocercoid invoking an IgE response. Parasitology 114: 263-271.

Kong Y, Yun DH, Cho SY, Sohn WM, Chung YB, Kang SY (2000) Differential expression of the $27 \mathrm{kDa}$ cathepsin Llike cysteine protease in developmental stages of Spirometra erinacei. Korean J Parasitol 38: 195-199. 